\title{
DIFFERENTIABILITY OF PERTURBED SEMIGROUPS AND DELAY SEMIGROUPS
}

\author{
CHARLES J. K. BATTY \\ St. John's College \\ Oxford OX1 3JP, Great Britain \\ E-mail: charles.batty@sjc.ox.ac.uk
}

\begin{abstract}
Suppose that $A$ generates a $C_{0}$-semigroup $T$ on a Banach space $X$. In $1953 \mathrm{R}$. S. Phillips showed that, for each bounded operator $B$ on $X$, the perturbation $A+B$ of $A$ generates a $C_{0}$-semigroup on $X$, and he considered whether certain classes of semigroups are stable under such perturbations. This study was extended in 1968 by A. Pazy who identified a condition on the resolvent of $A$ which is sufficient for the perturbed semigroups to be immediately differentiable. However, M. Renardy showed in 1995 that immediate differentiability is not stable under bounded perturbations.

We give a survey account of the partial answers already given to the question of differentiability of perturbed semigroups. Furthermore, we show that Pazy's condition is necessary, as well as sufficient, if one adds a natural requirement of uniformity for the differentiability of the perturbed semigroups. We also present an account of the corresponding theory for delay semigroups associated with $A$, based on an earlier paper of ours but with improved formulation. The necessary and sufficient condition for eventual differentiability of the delay semigroups is that the resolvent of $A$ should have polynomial decay on vertical lines. We also give a brief account of the consequences for asymptotics of individual mild solutions of abstract Cauchy problems and delay differential equations.
\end{abstract}

1. Background. Let $A$ be the generator of a $C_{0}$-semigroup $T=\{T(t): t \geq 0\}$ on a complex Banach space $X$, and let $B$ be a bounded linear operator on $X$. Phillips [21] showed that $A+B$ generates a $C_{0}$-semigroup $S_{B}$ on $X$ (see [1], [13] or [20] for general background information on $C_{0}$-semigroups and this result in particular). He raised the question which properties of semigroups are "stable" in the sense that if $T$ has the property then $S_{B}$ also has it for all $B \in \mathcal{B}(X)$. He argued that the stable properties are more significant, on both practical and mathematical grounds, than other properties.

2000 Mathematics Subject Classification: Primary 47D06; Secondary 34K12, 47A10, 93C15.

Key words and phrases: semigroup, immediately differentiable, eventually differentiable, bounded perturbation, stable property, resolvent, delay equation, asymptotics, growth bound.

The paper is in final form and no version of it will be published elsewhere. 
In particular, Phillips established stability of the class of holomorphic semigroups, i.e. those semigroups $T$ which extend to holomorphic mappings on a sector in $\mathbb{C}$ containing $(0, \infty)$ with appropriate continuity properties at 0 . This class includes many semigroups generated by elliptic differential operators.

THEOREM 1.1 (Phillips [21]). If $T$ is holomorphic, then $S_{B}$ is holomorphic whenever $B \in$ $\mathcal{B}(X)$.

The proof of Theorem 1.1 may be found in [1, Theorem 3.7.23], [13, Proposition III.1.12] and [20, Corollary 3.2.2]. It relies on the following two standard facts. Here and later, we write $R(\lambda, A)$ for $(\lambda I-A)^{-1}$ when $\lambda$ belongs to the resolvent set $\rho(A)$ of $A$.

1. A densely defined operator $A$ generates a holomorphic $C_{0}$-semigroup if and only if there exist $r>0$ and $c>0$ such that $\lambda \in \rho(A)$ and $\|\lambda R(\lambda, A)\| \leq c$ whenever $\operatorname{Re} \lambda>0$ and $|\lambda|>r$.

2. If $\lambda \in \rho(A)$ and $\|B\|\|R(\lambda, A)\|<1$, then $\lambda \in \rho(A+B)$ and

$$
\|R(\lambda, A+B)\|=\left\|R(\lambda, A) \sum_{n=0}^{\infty}(B R(\lambda, A))^{n}\right\| \leq \frac{\|R(\lambda, A)\|}{1-\|B\|\|R(\lambda, A)\|} .
$$

Phillips also addressed stability of norm-continuity of semigroups. A $C_{0}$-semigroup $T$ is said to be eventually norm-continuous if there exists $t_{0} \geq 0$ such that $T$ is continuous from $\left(t_{0}, \infty\right)$ to $\mathcal{B}(X)$ with respect to the operator norm; $T$ is said to be immediately norm-continuous if one can take $t_{0}=0$. Many semigroups associated with delay equations are eventually, but not immediately, norm-continuous (see Section 4).

Theorem 1.2 (Phillips [21]). If $T$ is immediately norm-continuous, then $S_{B}$ is immediately norm-continuous whenever $B \in \mathcal{B}(X)$.

The proof of Theorem 1.2 is given in [13, Theorem III.1.16]. In contrast to Theorem 1.1, it does not rely on a characterization of the generators of immediately normcontinuous semigroups. Indeed, no characterization is known even now. A simple application of the Riemann-Lebesgue Lemma shows that if $T$ is immediately norm-continuous then

$$
\lim _{|s| \rightarrow \infty}\|R(a+i s, A)\|=0
$$

for $a>\omega_{0}(T)$, the exponential growth bound of $T$ (see Section 5$)$. Putting $B=\left(a-a^{\prime}\right) I$ in (1.1) shows that

$$
\left\|R\left(a^{\prime}+i s, A\right)\right\| \leq 2\|R(a+i s, A)\| \quad \text { if } \quad\left|a^{\prime}-a\right| \leq(2\|R(a+i s, A)\|)^{-1},
$$

so (1.2) is independent of $a$, and for simplicity we shall take $a=0$ in later results.

It is known that (1.2) is equivalent to immediate norm-continuity of $T$ if $X$ is a Hilbert space (see [1, Theorem 3.13.2], [13, Theorem II.4.20]). However the following question is still open in Banach spaces.

Open Question 1. If A generates a $C_{0}$-semigroup $T$ on a Banach space and (1.2) holds, is $T$ immediately norm-continuous?

It follows easily from (1.1) that (1.2) is stable under bounded perturbations, so a positive answer to Open Question 1 would provide an alternative proof of Theorem 1.2. 
On the other hand, eventual norm-continuity is unstable.

ExAmple $1.3([21])$. Let $X=L^{1}(0,1)$ and define

$$
\begin{aligned}
(T(t) f)(s) & = \begin{cases}f(s+t) & (s+t \leq 1), \\
0 & (s+t>1),\end{cases} \\
(B f)(s) & = \begin{cases}f\left(s-\frac{1}{2}\right) & \left(s>\frac{1}{2}\right), \\
0 & \left(s \leq \frac{1}{2}\right) .\end{cases}
\end{aligned}
$$

Then $T(t)=0$ for $t \geq 1$, but $S_{B}$ is not eventually norm-continuous. We refer the reader to $[21$, Theorem 5.2] for a proof of this.

Stability of properties of semigroups was considered again by Pazy [19]. He showed that immediate compactness of a semigroup is stable, and he also considered the question whether immediate differentiability is stable. It is this question which is the main subject of this article.

2. Differentiability of semigroups. Let $T=\{T(t): t \geq 0\}$ be a $C_{0}$-semigroup, with generator $A$, on a complex Banach space $X$. For $x \in X$, let

$$
u_{x}(t)=T(t) x \quad(t \geq 0) .
$$

For $x \in D(A), u=u_{x}$ is a classical solution of the abstract Cauchy problem

$$
u^{\prime}(t)=A u(t) \quad(t \geq 0), \quad u(0)=x .
$$

For $x \in X, u_{x}$ is the unique mild solution of (ACP); that is, $u=u_{x}$ satisfies

$$
\int_{0}^{t} u(s) d s \in D(A) \quad \text { and } \quad u(t)=x+A\left(\int_{0}^{t} u(s) d s\right) \quad(t \geq 0) .
$$

Thus differentiability of the orbits of a semigroup corresponds to mild solutions becoming classical solutions.

The following proposition is elementary.

Proposition 2.1. Let $t_{0} \geq 0$. The following are equivalent:

(i) For each $x \in X$, the function $u_{x}$ is differentiable on $\left(t_{0}, \infty\right)$;

(ii) For each $t>t_{0}, T(t)$ maps $X$ into $D(A)$;

(iii) The function $T$ is differentiable on $\left(t_{0}, \infty\right)$ with respect to the operator norm.

When these conditions are satisfied, the derivative $T^{\prime}(t)=A T(t)$ for $t>t_{0}$. Moreover, for $n \geq 1, T$ is $n$-times differentiable on $\left(n t_{0}, \infty\right)$ and $T^{(n)}(t)=A^{n} T(t)$ for $t>n t_{0}$.

The $C_{0}$-semigroup $T$ is said to be eventually differentiable if the equivalent conditions of Proposition 2.1 are satisfied for some $t_{0} ; T$ is immediately differentiable if the conditions are satisfied for $t_{0}=0$. Clearly any holomorphic semigroup is immediately differentiable.

The following simple question seems to be open. It is not directly relevant to the rest of this article, but a solution would be of some interest.

Open Question 2. Suppose that, for each $x \in X$, there exists $t_{x}$ such that $u_{x}$ is differentiable on $\left(t_{x}, \infty\right)$. Is $T$ eventually differentiable? 
Pazy [19] characterized the generators of eventually, or immediately, differentiable semigroups as follows. For $\beta>0$ and $c \in \mathbb{R}$, let

$$
D_{\beta, c}=\{\lambda \in \mathbb{C}: \operatorname{Re} \lambda \geq c-\beta \log |\operatorname{Im} \lambda|\} .
$$

For $\beta>0, \kappa>0$ and $m \geq 0$, consider the following condition:

$\left(\mathrm{Q}_{\beta \kappa m}\right)$ There exists $c$ such that $D_{\beta, c} \subseteq \rho(A)$ and $\|R(\lambda, A)\| \leq \kappa|\operatorname{Im} \lambda|^{m} \quad\left(\lambda \in D_{\beta, c}\right)$.

Theorem 2.2 (Pazy [19]). The $C_{0}$-semigroup $T$ is eventually (resp., immediately) differentiable if and only if, for some (resp., all) $\beta>0$, there exist $\kappa, m$ such that $\left(\mathrm{Q}_{\beta \kappa m}\right)$ holds. More precisely, if $T$ is differentiable on $\left(t_{0}, \infty\right)$, then $\left(\mathrm{Q}_{\beta \kappa 1}\right)$ holds for some $\kappa$ whenever $\beta \in\left(0,1 / t_{0}\right)$. If $\left(\mathrm{Q}_{\beta \kappa m}\right)$ holds, then $T$ is differentiable on $((m+2) / \beta, \infty)$.

This characterization seems rather curious in two respects. Firstly, the regions $D_{\beta, c}$ are bounded by curves with exponential growth; secondly, polynomial growth of the resolvent is allowed, but one can always arrange linear growth. However, the resolvent of an eventually norm-continuous semigroup may not be bounded in any of the regions $D_{\beta, c}$. Indeed, this occurs for the shift semigroup on $C[0,1]$ given by

$$
(T(t) f)(s)= \begin{cases}f(s+t) & (s+t \leq 1) \\ f(1) & (s+t>1) .\end{cases}
$$

This is essentially due to Pazy [19, p. 1136].

The proof of Theorem 2.2 can be found in [20, Theorem 2.4.7]. It is based on the following formulae:

$$
\begin{aligned}
A T(t) & =\frac{1}{2 \pi i} \int_{\Gamma} \lambda e^{\lambda t} R(\lambda, A) d \lambda \\
\lambda e^{\lambda t} R(\lambda, A) & =A T(t) R(\lambda, A)+T(t)+\lambda e^{\lambda t} \int_{0}^{t} e^{-\lambda s} T(s) d s .
\end{aligned}
$$

In (2.1), it is assumed that $\left(\mathrm{Q}_{\beta \kappa m}\right)$ holds and then (2.1) is valid for a suitable contour $\Gamma$. The formula (2.2) is valid for any $C_{0}$-semigroup $T$ and any $\lambda \in \rho(A)$.

When $T$ is immediately differentiable, these formulae lead to estimates for $\|A T(t)\|$ in terms of $\|R(\lambda, A)\|$, and vice versa. In particular, (2.2) provides the estimate

$$
\|R(\lambda, A)\| \leq \frac{M_{t}\left(1+|\lambda| e^{\operatorname{Re} \lambda t} \int_{0}^{t} e^{-\operatorname{Re} \lambda s} d s\right)}{|\lambda| e^{\operatorname{Re} \lambda t}-\|A T(t)\|} \quad \text { if } \quad\|A T(t)\|<|\lambda| e^{\operatorname{Re} \lambda t},
$$

where $M_{t}=\sup \{\|T(s)\|: 0 \leq s \leq t\}$. If one knows estimates for $\|A T(t)\|$, one can then choose $t$, depending on $\lambda$, in such a way as to provide the sharpest estimates for $\|R(\lambda, A)\|$. We now recall two cases in the literature where this has been done. These involve simpler estimates for the resolvent than in Theorem 2.2.

Firstly, Yosida [26] (see also [27]) considered the case when there exist $\alpha>0$ and $c$ such that

$$
\|A T(t)\| \leq c e^{\alpha / t} \quad \text { for all sufficiently small } t>0 .
$$

Putting $\lambda=i s$ where $s$ is real, and $t=\kappa(\log |s|)^{-1}$ for $\kappa>\alpha$ in (2.3) leads to

$$
\|R(i s, A)\| \leq \frac{\gamma}{\log |s|} \quad \text { whenever }|s| \text { is sufficiently large, }
$$

provided that $\gamma>\alpha M_{0+}$, where $M_{0+}=\lim \sup _{t \rightarrow 0+}\|T(t)\|$. 
Conversely, the validity of (2.5) for some $\gamma$ implies, via (1.3), that $\left(\mathrm{Q}_{\beta \kappa 0}\right)$ holds when $\beta<1 / \gamma$, and then it follows from Theorem 2.2 that $T$ is eventually differentiable, and immediately differentiable if (2.5) holds for each $\gamma>0$. However, even if $T$ is immediately differentiable, (2.5) does not imply any estimate for $\|A T(t)\|$ (see [15]).

Secondly, Crandall and Pazy [7] considered the more special case when there exist $\alpha \geq 1$ and $c$ such that

$$
\|A T(t)\| \leq c t^{-\alpha} \quad \text { for all sufficiently small } t>0 .
$$

Putting $\lambda=i s$ and $t=\kappa|s|^{-1 / \alpha}$ for $\kappa>c^{1 / \alpha}$ in (2.3) shows that

$$
\|R(i s, A)\| \leq \frac{\gamma}{|s|^{1 / \alpha}} \quad \text { whenever }|s| \text { is sufficiently large, }
$$

for some $\gamma$. On the other hand, the formula (2.1) was used in [7] to show that (2.7) implies that there exists $c^{\prime}$ such that

$$
\|A T(t)\| \leq c^{\prime} t^{-(2 \alpha-1)} \quad \text { for all sufficiently small } t>0 .
$$

A moment inequality was used in [12] to improve this to

$$
\|A T(t)\| \leq c_{\varepsilon}^{\prime} t^{-(\alpha+\varepsilon)} \quad \text { for all sufficiently small } t>0,
$$

for each $\varepsilon>0$. The class of semigroups satisfying (2.6), or equivalently a resolvent estimate of the form (2.7), will be considered again in Section 4.

A third, more general, case when an estimate for $\|A T(t)\|$ implies a resolvent estimate will be considered in Proposition 3.8.

3. Bounded perturbations. In the spirit of Phillips, Pazy [19] asked the following question.

PAZY'S QUESTION, ORIGINAL VERSION. Is the class of immediately differentiable $C_{0}$ semigroups stable under bounded perturbations?

This question was open for a long time, until Renardy showed that the answer is negative.

EXAMPLE 3.1 ([22]). There is an immediately differentiable semigroup $T$ on a Hilbert space, and a bounded operator $B$ such that $S_{B}$ is not eventually differentiable. In the example given in [22], $A$ is a direct sum of $n \times n$ matrices $A_{n}(n=1,2, \ldots)$ chosen in such a way that $\left(\mathrm{Q}_{\beta \kappa 1}\right)$ holds for $A_{n}$ uniformly in $n$, but $\left\|R\left(\lambda_{n}, A_{n}\right)\right\| \geq 1$ for a sequence of points $\lambda_{n} \in \mathbb{C}$ such that $\left\{\lambda_{n}: n \geq 1\right\}$ is not contained in any set of the form $D_{\beta, c}$ for $\beta>0$. Then there exist $n \times n$ matrices $B_{n}$ of rank one such that $\left\|B_{n}\right\| \leq 1$ and $\lambda_{n}$ is an eigenvalue of $A_{n}+B_{n}$. Taking $B$ to be the direct sum of the perturbations $B_{n}$ provides the counterexample. We refer the reader to [22] for the details.

So Pazy's question can be modified to the following.

PAZY'S QUESTION, SECOND VERSION. For which semigroups $T$ is it true that $S_{B}$ is immediately (or eventually) differentiable for all $B \in \mathcal{B}(X)$ ?

It is this question, or a slight variant of it, which we shall discuss in this section. An answer in terms of properties of $A$ is more useful for applications than an answer 
involving conditions on $T$. Pazy himself had deduced a positive result in this direction from his criterion for differentiability of semigroups.

Let $\beta>0$, and consider the following condition:

$$
\text { There exists } c \text { such that } D_{\beta, c} \subseteq \rho(A) \text { and } \lim _{\substack{\lambda \in D_{\beta, c} \\|\operatorname{Im} \lambda| \rightarrow \infty}}\|R(\lambda, A)\|=0 .
$$

It is clear that $\left(\mathrm{P}_{\beta}\right)$ implies $\left(\mathrm{Q}_{\beta \kappa 0}\right)$ for every $\kappa>0$. On the other hand, if there exists $\beta$ such that $\left(\mathrm{Q}_{\beta \kappa 0}\right)$ holds for every $\kappa>0$, then $\left(\mathrm{P}_{\beta^{\prime}}\right)$ holds whenever $\beta^{\prime} \in(0, \beta)$ (see the proof of Corollary 3.6 below). It is immediate from (1.1) that $\left(\mathrm{P}_{\beta}\right)$ is invariant under bounded perturbations of $A$ and then Theorem 2.2 implies the following.

Theorem 3.2 (Pazy [19]). If A satisfies $\left(\mathrm{P}_{\beta}\right)$ for some $\beta>0$, then $S_{B}$ is differentiable on $(2 / \beta, \infty)$ for every $B \in \mathcal{B}(X)$. In particular, if $A$ satisfies $\left(\mathrm{P}_{\beta}\right)$ for every $\beta>0$, then $S_{B}$ is immediately differentiable.

A more precise version is the following.

Proposition 3.3. If A satisfies $\left(\mathrm{Q}_{\beta \kappa 0}\right)$ for some $\beta>0$ and $\kappa>0$, then $S_{B}$ is differentiable on $(2 / \beta, \infty)$ whenever $\|B\|<\kappa^{-1}$.

In the situation of Theorem 3.2 and Proposition 3.3, the eventual differentiability of $S_{B}$ is uniform with respect to $B$ in both of the following respects:

(U1) There exists $t_{0}$, independent of $B$, such that $S_{B}$ is differentiable on $\left(t_{0}, \infty\right)$ for all relevant $B$;

(U2) For each relevant $\gamma$, there exists $g_{\gamma}:\left(t_{0}, \infty\right) \rightarrow \mathbb{R}$ such that $\left\|(A+B) S_{B}(t)\right\| \leq g_{\gamma}(t)$ for all $t>t_{0}$ and for all $B$ with $\|B\| \leq \gamma$.

In (U2), $\gamma$ is arbitrary in the context of Theorem 3.2, but there is a restriction that $\gamma<\kappa^{-1}$ in the context of Proposition 3.3.

The uniformity (U1) is explicit in the statements of Theorem 3.2 and Proposition 3.3, with $t_{0}=2 / \beta$. The uniformity (U2) can be seen either by examining the proofs of those results or by applying the results themselves to direct sums of copies of $T$. We shall show in Theorem 3.5 and its corollaries that converses of Theorem 3.2 and Proposition 3.3 hold if we assume both of these uniformities. It is possible that either or both of these uniformities may be automatic; see Open Question 3 below.

In order to give the converses of Pazy's results Theorem 3.2 and Proposition 3.3, we need the following elementary lemma.

Lemma 3.4. Let $\lambda \in \mathbb{C}$ and $\gamma>0$. The following are equivalent:

(i) $\lambda \in \rho(A)$ and $\|R(\lambda, A)\| \leq \gamma^{-1}$;

(ii) $\lambda \in \rho(A+B)$ whenever $B \in \mathcal{B}(X)$ and $\|B\|<\gamma$;

(iii) $\lambda \in \rho(A+B)$ whenever $B$ is of rank one and $\|B\|<\gamma$.

Proof. It is immediate from (1.1) that (i) implies (ii), and it is trivial that (ii) implies (iii).

Suppose that (iii) holds. Then $\lambda \in \rho(A)$. Suppose that $\|R(\lambda, A)\|>\gamma^{-1}$. Then there exists $x \in X$ with $\|x\|=1$ and $\|R(\lambda, A) x\|>\gamma^{-1}$, and there exists $\psi \in X^{*}$ with $\|\psi\|<\gamma$ and $\psi(R(\lambda, A) x)=1$. Define $B y=\psi(y) x(y \in X)$. Then $B$ is rank one, $\|B\|<\gamma$ and $(\lambda-(A+B)) R(\lambda, A) x=0$. This would contradict (iii). This proves that (iii) implies (i). 
The following can be found in [4], but its significance was not made explicit there.

THEOREM 3.5. Suppose that there exist $t_{0} \geq 0$ and $\gamma>0$ such that $S_{B}$ is differentiable on $\left(t_{0}, \infty\right)$ whenever $B$ is an operator of rank one with $\|B\| \leq \gamma$, and the differentiability is uniform in the sense of (U2). Then $\left(\mathrm{Q}_{\beta, \gamma^{-1}, 0}\right)$ holds whenever $0<\beta<1 / t_{0}$.

Proof. By Theorem 2.2, there exists $c$ such that $D_{\beta, c} \subseteq \rho(A+B)$ whenever $B$ is of rank one and $\|B\| \leq \gamma$. By Lemma 3.4, $\|R(\lambda, A)\| \leq \gamma^{-1}$ whenever $\lambda \in D_{\beta, c}$.

Corollary 3.6. Suppose that $S_{B}$ is differentiable on $\left(t_{0}, \infty\right)$ for each operator $B$ of rank one on $X$, and (U2) holds for each $\gamma>0$. Then $\left(\mathrm{P}_{\beta}\right)$ holds whenever $0<\beta<1 / t_{0}$.

Proof. Given $\beta$ with $0<\beta<1 / t_{0}$, choose $\beta^{\prime}$ such that $\beta<\beta^{\prime}<1 / t_{0}$. By Theorem 3.5, there exist $c_{n}$ such that $D_{\beta^{\prime}, c_{n}} \subseteq \rho(A)$ and $\|R(\lambda, A)\| \leq 1 / n$ whenever $\lambda \in D_{\beta^{\prime}, c_{n}}$. Let $c=c_{1}$. Then $D_{\beta, c} \subseteq D_{\beta^{\prime}, c_{1}} \subseteq \rho(A)$. If $\lambda \in D_{\beta, c}$ and $|\operatorname{Im} \lambda|>\exp \left(\left(c_{n}-c\right) /\left(\beta^{\prime}-\beta\right)\right)$, then $\lambda \in D_{\beta^{\prime}, c_{n}}$, so $\|R(\lambda, A)\| \leq 1 / n$. Thus $\left(\mathrm{P}_{\beta}\right)$ holds.

Corollary 3.7. Suppose that $S_{B}$ is immediately differentiable for each operator $B$ of rank one on $X$, and (U2) holds for each $\gamma>0$. Then $\left(\mathrm{P}_{\beta}\right)$ holds for every $\beta>0$.

We have now answered a third version of Pazy's question: in order that the semigroups $S_{B}$ should be eventually (resp., immediately) differentiable for all $B$ uniformly in the sense of (U1) and (U2), it is necessary and sufficient that the generator $A$ should satisfy the condition $\left(\mathrm{P}_{\beta}\right)$ for some (resp., all) $\beta>0$.

At least in the case of immediate differentiability, we think that the answer to the second version of Pazy's question is probably the same. Equivalently, we expect that the following question has a positive answer.

Open Question 3. Can the assumptions of uniformity (U2) be omitted in Theorem 3.5 and its corollaries?

In Theorem 3.5, Corollary 3.6 and Corollary 3.7, we have assumed that the uniformity (U1) holds, i.e., that $t_{0}$ is independent of $B$. We think it is unlikely that (U1) is automatic in the context of Theorem 3.5; indeed, we expect that there exists a generator $A$ such that, for each $\kappa>0$, there exists $\beta_{\kappa}>0$ such that $\left(\mathrm{Q}_{\beta_{\kappa} \kappa 0}\right)$ holds but $\beta_{\kappa}$ cannot be chosen independent of $\kappa$. Then $S_{B}$ would be eventually differentiable for every $B$ but it would not be possible to choose $t_{0}$ uniformly for $B$ of arbitrarily large norm.

The results above show that counterexamples to Pazy's original question, or the corresponding question for eventual differentiability, correspond almost exactly to examples where $\left(\mathrm{Q}_{\beta \kappa 1}\right)$ holds for some $\kappa$ (for some, or all, $\beta$ ), but $\left(\mathrm{Q}_{\beta \kappa 0}\right)$ fails for some $\kappa$. If Open Question 3 has a negative answer, such an example might not itself be a counterexample to Pazy's question, but it would provide a counterexample by means of a direct sum construction. Thus it was not chance that the shift semigroup $S$ on either $L^{1}[0,1]$ or $C[0,1]$ provided both the first example that eventual differentiability is not stable under bounded perturbations (see Example 1.3) and the first example that eventual differentiability does not imply $\left(\mathrm{Q}_{\beta \kappa 0}\right)[19, \mathrm{p} .1136]$. For an example of an immediately differentiable semigroup with such properties, we refer to Renardy's example [22] (see Example 3.1). It is not difficult to verify directly that Renardy's example fails to satisfy $\left(\mathrm{Q}_{\beta \kappa 0}\right)$. Then Corollary 3.6 implies that the rank one perturbations do not generate semigroups which are eventually 
differentiable uniformly in the sense (U2). In fact Renardy showed directly that there is an explicit bounded perturbation $B$ such that $S_{B}$ is not eventually differentiable. The operator $B$ is not of finite rank, but $A$ is constructed as a direct sum of operators $A_{n}$ on finite-dimensional spaces and $B$ is a direct sum of rank one perturbations of $A_{n}$. So Renardy's construction and the proof of Theorem 3.5 both depend on the same simple idea described in Lemma 3.4.

Now we discuss a result of Doytchinov, Hrusa and Watson [11]. They showed that if $T$ is immediately differentiable and there exist $c$ and $\alpha>0$ such that

$$
\|A T(t)\| \leq c t^{-\alpha / t} \quad \text { for all sufficiently small } t>0 \text {, }
$$

then $S_{B}$ is differentiable on $(\alpha, \infty)$, for every $B \in \mathcal{B}(X)$. Thus (U1) is satisfied, explicitly. Moreover, (U2) is also satisfied. This can be seen either from the proof in [11] or by applying the result of [11] to a direct sum. It follows from Corollary 3.6 that (3.1) implies $\left(\mathrm{P}_{\beta}\right)$ for $0<\beta<1 / \alpha$. We shall show directly that $(3.1)$ implies $\left(\mathrm{P}_{\beta}\right)$ for $0<\beta \leq 1 / \alpha$. In addition to the general illumination which this direct proof offers, in combination with Theorem 3.2 it provides an alternative means of establishing that (3.1) implies that $S_{B}$ is eventually differentiable (although this route only establishes differentiability for $t>2 \alpha$ rather than for $t>\alpha$ ). The proof in [11] did not use Theorem 2.2.

Proposition 3.8. Suppose that $T$ is immediately differentiable and (3.1) holds. Then $\left(\mathrm{P}_{1 / \alpha}\right)$ holds.

Proof. By Theorem 2.2, there exists $c$ such that $D_{\beta, c} \subseteq \rho(A)$. We may assume that $c>0$. Take $\lambda \in D_{\beta, c}$ with $\operatorname{Re} \lambda<-e$, and let

$$
a=-\operatorname{Re} \lambda, \quad t=\frac{\log a-\frac{1}{2} \log \log a}{a} .
$$

If $a$ is sufficiently large, then (3.1) gives

Hence,

$$
\|A T(t)\| \leq c\left(\frac{a}{\log a-\frac{1}{2} \log \log a}\right)^{\alpha a /\left(\log a-\frac{1}{2} \log \log a\right)} .
$$

$$
\begin{aligned}
& \log \left(\frac{\|A T(t)\|}{e^{\alpha a} e^{-a t}}\right) \\
& \leq \log c+\frac{\alpha a}{\log a-\frac{1}{2} \log \log a} \log \left(\frac{a}{\log a-\frac{1}{2} \log \log a}\right)-\alpha a+\log a-\frac{1}{2} \log \log a \\
& \leq \log c-\alpha a \frac{\log a-\frac{1}{2} \log \log a-\log \left(\frac{a}{\log a-\frac{1}{2} \log \log a}\right)}{\log a-\frac{1}{2} \log \log a}+\log a \\
& \leq \log c-\alpha a \frac{\log \left(\frac{\log a-\frac{1}{2} \log \log a}{(\log a)^{1 / 2}}\right)}{\log a}+\log a \\
& \leq \log c-\frac{\alpha a}{\log a}+\log a \leq-\log 2
\end{aligned}
$$


for all sufficiently large $a$. Thus if $a$ is sufficiently large and $|\operatorname{Im} \lambda| \geq e^{\alpha a}$, then

$$
\|A T(t)\| \leq \frac{1}{2} e^{\alpha a} e^{-a t} \leq \frac{1}{2}|\lambda| e^{-a t} .
$$

It then follows from (2.3) that

$$
\begin{aligned}
\|R(\lambda, A)\| & \leq M_{1}\left(\frac{1+|\lambda| e^{-a t} \frac{e^{a t}-1}{a}}{\frac{1}{2}|\lambda| e^{-a t}}\right) \\
& \leq 2 M_{1}\left(\frac{e^{a t}}{|\lambda|}+\frac{\frac{a}{(\log a)^{1 / 2}}-1}{a}\right) \\
& \leq 2 M_{1}\left(\frac{a}{(\log a)^{1 / 2} e^{\alpha a}}+\frac{1}{(\log a)^{1 / 2}}\right) .
\end{aligned}
$$

This shows that

$$
\lim _{\substack{\lambda \in D_{1 / \alpha, c} \\ \operatorname{Re} \lambda \rightarrow-\infty}}\|R(\lambda, A)\|=0 .
$$

Since $T$ is immediately norm-continuous, it follows from (1.2) and the subsequent discussion that

$$
\lim _{\substack{\operatorname{Re} \lambda>-\kappa \\|\operatorname{Im} \lambda| \rightarrow \infty}}\|R(\lambda, A)\|=0,
$$

for every $\kappa$. Thus $\left(P_{1 / \alpha}\right)$ is satisfied.

If one merely wishes to establish that $\left(\mathrm{P}_{\beta}\right)$ holds for $\beta<1 / \alpha$, then one may take $t=\frac{\gamma \log a}{a}$, where $\gamma<1$, in the proof above.

Renardy's construction was modified in [11, Theorem 2] to show that (3.1) is sharp as an estimate for $\|A T(t)\|$ which is sufficient to ensure that $S_{B}$ is eventually differentiable, so the estimate (3.1) is also sharp for the purposes of Proposition 3.8. Another modification of Renardy's example given in the proof of [11, Theorem 3] shows that (3.1) does not imply $\left(\mathrm{P}_{\beta}\right)$ for $\beta>16 / \alpha$. It is clear that the estimates there can be sharpened to replace $16 / \alpha$ by a smaller value, but we have not succeeded in establishing that $1 / \alpha$ is optimal in Proposition 3.8.

This article is mainly about conditions on $A$ which imply, or are implied by, the property that $S_{B}$ is immediately differentiable for all $B$. In applications one may be interested only in special operators $B \in \mathcal{B}(X)$, and then one seeks conditions on $A$ (or $T$ ) and $B$ which imply that $S_{B}$ is differentiable. One such result was obtained by Nagel and Piazzera [18, Theorem 6.5]. Here, we let

$$
\left(V_{B} f\right)(t)=\int_{0}^{t} T(t-s) B f(s) d s \quad(t \geq 0)
$$

for any strongly continuous function $f:[0, \infty) \rightarrow \mathcal{B}(X)$. Then $V_{B}$ is a linear operator on the vector space of all such functions $f$.

Theorem 3.9 (Nagel and Piazzera [18]). Suppose that there exists $n$ such that $V_{B}^{n} f$ is norm-differentiable from $[0, \infty)$ to $\mathcal{B}(X)$ whenever $f:[0, \infty) \rightarrow \mathcal{B}(X)$ is strongly continuous. If $T$ is eventually (resp., immediately) differentiable, then $S_{B}$ is eventually (resp., immediately) differentiable. 
4. Delay semigroups. Suppose that $A$ generates a $C_{0}$-semigroup $T$ on $X$ and $\Phi$ : $C([-1,0], X) \rightarrow X$ is a bounded linear operator. It is well known (see [13, Section VI.6], for a brief account; or any of [10], [14], [25] for fuller accounts with applications) that there is an associated delay semigroup $\left\{V_{\Phi}(t): t \geq 0\right\}$ on $C([-1,0], X)$ whose generator $B_{\Phi}$ is given by

$$
\begin{aligned}
D\left(B_{\Phi}\right) & =\left\{f \in C^{1}([-1,0], X): f(0) \in D(A) \text { and } f^{\prime}(0)=A f(0)+\Phi f\right\}, \\
B_{\Phi} f & =f^{\prime} .
\end{aligned}
$$

This semigroup is a tool for studying the delay differential equation

$$
u^{\prime}(t)=A u(t)+\Phi u_{t} \quad(t \geq 0), \quad u_{0}=f .
$$

Here, $u_{t}$ is the history function of $u$ given by $u_{t}(\theta)=u(t+\theta)(t \geq 0,-1 \leq \theta \leq 0)$, and $f$ is a given initial history. If $f \in D\left(B_{\Phi}\right)$, then there is a unique classical solution of (DDE) given by

$$
u(t)= \begin{cases}f(t) & (-1 \leq t \leq 0), \\ \left(V_{\Phi}(t) f\right)(0) & (t \geq 0) .\end{cases}
$$

More generally, for any $u \in C([-1,0], X)$, this formula defines the unique mild solution of (DDE); that is, $u$ satisfies $u_{0}=f$ and $\int_{0}^{t} u(s) d s \in D(A)$ and

$$
u(t)=u(0)+A\left(\int_{0}^{t} u(s) d s\right)+\int_{0}^{t} \Phi u_{s} d s
$$

for all $t \geq 0$.

The semigroup $V_{\Phi}$ has the following properties:

$$
\begin{aligned}
\left(V_{\Phi}(t) f\right)(\theta) & = \begin{cases}f(t+\theta) & \text { if } t+\theta \leq 0, \\
\left(V_{\Phi}(t+\theta)\right) f(0) & \text { if } t+\theta \geq 0,\end{cases} \\
\left(V_{\Phi}(t) f\right)(0) & =T(t) f(0)+\left(T * \Phi V_{\Phi}\right)(t) f \\
& =T(t) f(0)+\int_{0}^{t} T(t-s) \Phi V_{\Phi}(s) f d s .
\end{aligned}
$$

It follows easily from (4.3) that $V_{\Phi}$ is eventually norm-continuous if $T$ is immediately norm-continuous [13, Theorem VI.6.6]. However, $V_{\Phi}$ is not immediately norm-continuous, because it acts as a shift for $t+\theta \leq 0$.

The following elementary result shows that eventual differentiability of $V_{\Phi}$ corresponds exactly to the mild solutions of (DDE) becoming classical solutions after a time which is independent of the initial history $f$.

Proposition 4.1 ([4]). The following are equivalent:

(i) $V_{\Phi}$ is eventually differentiable;

(ii) There exists $t_{0} \geq 0$ such that, for each $f \in C([-1,0], X)$, the unique mild solution of (DDE) is a classical solution on $\left(t_{0}, \infty\right)$.

The question of characterizing generators of semigroups with the property that $V_{\Phi}$ is eventually differentiable for every $\Phi \in \mathcal{B}(C([-1,0], X), X)$ was addressed in [4]. The following solution is taken from results in [4] together with an earlier result from [7]. 
TheOREM 4.2. Let $A$ be the generator of an immediately differentiable $C_{0}$-semigroup $T$. The following are equivalent:

(i) There exist $\gamma$ and $\beta>0$ such that $\| R($ is, $A) \| \leq \gamma|s|^{-\beta}$ whenever $|s|$ is sufficiently large;

(ii) There exist $c$ and $\alpha>0$ such that $\|A T(t)\| \leq c t^{-\alpha}$ whenever $0<t \leq 1$;

(iii) $V_{\Phi}$ is eventually differentiable whenever $\Phi \in \mathcal{B}(C([-1,0], X), X)$;

(iv) $V_{\Phi}$ is eventually differentiable when $\Phi f=f(-1)$.

We give only an outline of the proof here, referring the reader to [4] for details. As noted in Section 2, it was shown in [7] (see also [12]) that (i) implies (ii) and conversely. It is trivial that (iii) implies (iv).

The proof that (i) implies (iii) uses Theorem 2.2 together with the following well known description of the resolvent of $V_{\Phi}$ [13, Proposition VI.6.7]. Let $\lambda \in \mathbb{C}$. For $x \in X$, define $\varepsilon_{\lambda} \otimes x \in C([-1,0], X)$ by $\left(\varepsilon_{\lambda} \otimes x\right)(\theta)=e^{\lambda \theta} x$. Define bounded linear operators $\Phi_{\lambda}$ on $X$ and $H_{\lambda}$ on $C([-1,0], X)$ by

$$
\begin{aligned}
\Phi_{\lambda}(x) & =\Phi\left(\varepsilon_{\lambda} \otimes x\right), \\
\left(H_{\lambda} f\right)(\theta) & =\int_{\theta}^{0} e^{\lambda(\theta-\tau)} f(\tau) d \tau .
\end{aligned}
$$

Now $\lambda \in \rho\left(B_{\Phi}\right)$ if and only if $\lambda \in \rho\left(A+\Phi_{\lambda}\right)$ and then

$$
R\left(\lambda, B_{\Phi}\right) f=\varepsilon_{\lambda} \otimes\left(R\left(\lambda, A+\Phi_{\lambda}\right)\left(f(0)+\Phi H_{\lambda} f\right)\right)+H_{\lambda} f .
$$

For appropriate $\lambda$, one can use (1.1) to show that $\lambda \in \rho\left(A+\Phi_{\lambda}\right)$ and to estimate $\left\|R\left(\lambda, A+\Phi_{\lambda}\right)\right\|$. Then one can show that $B_{\Phi}$ satisfies $\left(\mathrm{Q}_{1 / \alpha, \kappa, 1}\right)$ for some $\kappa$ and Theorem 3.2 implies (iii).

The proof that (iv) implies (ii) uses the following representation of the mild solutions of (DDE) in the case when $\Phi f=f(-1)$ :

$$
u(n+t)=\sum_{r=0}^{n} \frac{t^{r}}{r !} T(t) u(n-r)+\frac{1}{n !} \int_{0}^{t} s^{n} T(s) g(t-s) d s \quad(n \in \mathbb{N}, t \in[0,1]) .
$$

The assumption in Theorem 4.2 that $T$ is immediately differentiable is used only in the proof that (iv) implies (i). Indeed, either (i) or (ii) implies that $T$ is immediately differentiable.

Open Question 4. Does condition (iv) (or (iii)) of Theorem 4.2 imply that $T$ is immediately differentiable?

It is remarkable that in Theorem 4.2, the differentiability of one particular delay semigroup associated with $A$ implies the eventual differentiability of all of them, even uniformly for $\|\Phi\| \leq \gamma$ for any $\gamma$. This is one reason for thinking that Open Question 3 should have a positive answer.

Delay semigroups provide further examples where eventual differentiability is unstable under bounded perturbations. Let $A$ be the generator of an eventually differentiable $C_{0^{-}}$ semigroup and consider the delay semigroup $V_{0}$ corresponding to $\Phi=0$. It follows from Proposition 4.1 that this semigroup is eventually differentiable and from (4.4) that its generator $B_{0}$ satisfies $\left\|R\left(\lambda, B_{0}\right)\right\| \geq e^{-\operatorname{Re} \lambda}$. Thus $B_{0}$ never satisfies $\left(\mathrm{Q}_{\beta \kappa 0}\right)$ or $\left(\mathrm{P}_{\beta}\right)$ for $\beta>0$ and $\kappa<1$. By Theorem 3.5 there are bounded perturbations of $B_{0}$ which generate 
semigroups which are not eventually differentiable uniformly in the sense (U2). Then a direct sum construction if necessary provides an example. Note that when $X=\mathbb{C}$ and $A=0, V_{0}$ is the shift semigroup on $C[-1,0]$ so this construction generalizes $[19$, p. 1136].

REMARK 4.3. There is a theory of delay semigroups based on $L^{p}$-spaces $(1 \leq p<\infty)$ (see [24], [2], [3], for example). In many applications $\Phi$ is not bounded from $L^{p}([-1,0], X)$ to $X$, but sometimes $\Phi$ is bounded from $C([-1,0], X)$ to $X$. Suppose that $\Phi$ is bounded from $C([-1,0], X)$ to $X$ and that there is an associated delay semigroup $V_{\Phi}$ on the space $X \times L^{p}([-1,0], X)$. For example, this is true whenever $\Phi$ is of the form $\Phi f=\int_{-1}^{0} d \eta f$ for some function $\eta:[-1,0] \rightarrow \mathcal{B}(X)$ of bounded variation [2, Example 3.4], [16, Theorem 1.1]. In this context, Theorem 4.2 remains valid.

5. Asymptotics. Suitable regularity of a $C_{0}$-semigroup $T$ has the important consequence that the exponential growth bound $\omega_{0}(T)$ of $T$ is determined by the spectral bound $s(A)$ of $A$. Here,

$$
\begin{aligned}
\omega_{0}(T) & =\inf \left\{\omega \in \mathbb{R}: \sup _{t \geq 0}\left\|e^{-\omega t} T(t)\right\|<\infty\right\}, \\
s(A) & =\sup \{\operatorname{Re} \lambda: \lambda \in \sigma(A)\} .
\end{aligned}
$$

It is well known that $s(A) \leq \omega_{0}(T)$ in general and that $s(A)=\omega_{0}(T)$ if $T$ is eventually norm-continuous [13, Corollary IV.3.11] (a more general result can be found in [17, Corollary 1.4]).

For $x \in X$, let $u_{x}(t)=T(t) x(t \geq 0)$. The Laplace transform is given by $\widehat{u_{x}}(\lambda)=$ $R(\lambda, A) x$ for $\operatorname{Re} \lambda>\omega_{0}(T)$. Moreover,

where

$$
\begin{aligned}
\omega_{0}(T) & =\sup \left\{\omega_{0}\left(u_{x}\right): x \in X\right\}, \\
s(A) & =\sup \left\{\operatorname{hol}\left(\widehat{u_{x}}\right): x \in X\right\},
\end{aligned}
$$

$$
\begin{aligned}
\omega_{0}(u) & =\inf \left\{\omega \in \mathbb{R}: \sup _{t \geq 0}\left\|e^{-\omega t} u(t)\right\|<\infty\right\}, \\
\operatorname{hol}(\hat{u}) & =\inf \{\omega \in \mathbb{R}: \hat{u} \text { has a holomorphic extension to }\{\operatorname{Re} \lambda>\omega\}\}
\end{aligned}
$$

(see $[1$, Sections 1.4, 5.1]). This raises the following question.

Open Question 5. Is it true that $\omega_{0}\left(u_{x}\right)=\operatorname{hol}\left(\widehat{u_{x}}\right)$ for each initial value $x \in X$, if $T$ is eventually norm-continuous?

We do not know the complete answer to this question. If $T$ is immediately normcontinuous, then the equality $\omega_{0}\left(u_{x}\right)=\operatorname{hol}\left(\widehat{u_{x}}\right)$ holds for $x \in D(A)$ (see Theorem 5.2(2) below). If $T$ is holomorphic, the equality holds for $x \in X$ by general theory of Laplace transforms [1, Theorems 2.6.2, 5.1.2]. The following more general positive answer is obtained from the theory of the non-analytic growth bound introduced by Blake [6] and developed in [5].

TheOREM 5.1 ([5, Theorem 5.7]). If $T$ is eventually differentiable, then $\omega_{0}\left(u_{x}\right)=$ $\operatorname{hol}\left(\widehat{u_{x}}\right)$ for all $x \in X$.

We can ask the same question about mild solutions $u$ of (DDE), i.e., functions of the form $u(t)=\left(V_{\Phi}(t) f\right)(0)(t \geq 0)$. The Laplace transform of $u$ is then given by

$$
\left.\hat{u}(\lambda)=\left(R\left(\lambda, B_{\Phi}\right) f\right)(0)=R\left(\lambda, A+\Phi_{\lambda}\right)\left(f(0)+\Phi H_{\lambda} f\right)\right) .
$$


Theorem 5.2 ([4, Theorems 3.2, 3.3]).

1. Suppose that there exist $\beta>0, b>0$ and $c>0$ such that is $\in \rho(A)$ and $\|R(i s, A)\| \leq c|s|^{-\beta}$ whenever $s \in \mathbb{R}$ and $|s|>b$. Then $\omega_{0}(u)=\operatorname{hol}(\hat{u})$ for each mild solution $u$ of (DDE).

2. Suppose that $\lim _{|s| \rightarrow \infty}\|R(a+i s, A)\|=0$ for some $a>\omega_{0}(T)$. Then $\omega_{0}(u)=\operatorname{hol}(\hat{u})$ for each classical solution $u$ of (DDE).

The first part of Theorem ?? follows quickly from Theorem 4.2 and Theorem 5.1. The second part follows from the description of the resolvent of $B_{\Phi}$ given in Section 4 and results in [5]. We refer the reader to [4] for the details.

6. Unbounded perturbations. There are various applications which can be modelled by semigroups generated by $A+B$ where $A$ is well understood and $B$ is an unbounded perturbation, or by a delay differential equation of the form (DDE) where the delay operator $\Phi$ is an unbounded operator, but $B$ or $\Phi$ is small with respect to $A$ in some sense. For example, $A$ may be a second-order elliptic differential operator and $B$ or $\Phi$ may involve first-order derivatives. Thus it is important for applications to allow unbounded perturbations or delay operators, and to be able to establish regularity of the solutions, but there is only a limited theory mainly confined to the case when $A$ generates a holomorphic semigroup.

There are several generation theorems for unbounded perturbations; a rather comprehensive survey account can be found in [13, Sections III.2, III.3]. Some of these theorems extend Theorem 1.1 by establishing that suitable unbounded perturbations of holomorphic semigroups are holomorphic, but otherwise little is known about differentiability of semigroups generated by unbounded perturbations. When $A$ generates a holomorphic semigroup and $\Phi$ is an unbounded delay operator satisfying some rather restrictive conditions, there are a few results showing that there is an associated delay semigroup which is eventually differentiable (see [8], [9], [23]) but there is as yet not an extensive general theory of this topic.

AdDed Note. Since this article was written, considerable progress has been made with the Open Questions 1, 2 and 3. Tamás Mátrai has constructed an example of a $C_{0^{-}}$ semigroup $T$ for which (1.2) holds but $T$ is not immediately norm-continuous, thereby giving a negative answer to Open Question 1. Positive answers to Open Question 2 and a slight variant of Open Question 3 have been obtained as a result of work of Philippa Iley (the variation is that it is assumed that $S_{B}$ is eventually or immediately differentiable for each compact operator $B$ instead of just for rank one operators).

\section{References}

[1] W. Arendt, C. J. K. Batty, M. Hieber and F. Neubrander, Vector-Valued Laplace Transforms and Cauchy Problems, Birkhäuser, Basel, 2001.

[2] A. Bátkai and S. Piazzera, Semigroups and linear partial differential equations with delay, J. Math. Anal. Appl. 264 (2001), 1-20. 
[3] A. Bátkai and S. Piazzera, Semigroups for Delay Equations in $L^{p}$-phase Spaces, A. K. Peters, Wellesley, 2006.

[4] C. J. K. Batty, Differentiability and growth bounds of solutions of delay equations, J. Math. Anal. Appl. 299 (2004), 133-146.

[5] C. J. K. Batty, M. D. Blake and S. Srivastava, A non-analytic growth bound for Laplace transforms and semigroups of operators, Integral Equations Operator Theory 45 (2003), $125-154$.

[6] M. D. Blake, Asymptotically norm-continuous semigroups of operators, DPhil thesis, Oxford, 1999.

[7] M. G. Crandall and A. Pazy, On the differentiability of weak solutions of a differential equation in Banach space, J. Math. Mech. 18 (1968/69), 1007-1016.

[8] G. Di Blasio, Differentiability of the solution semigroup for delay differential equations, in: Evolution Equations, G. R Goldstein, R. Nagel and S. Romanelli (eds.), Marcel Dekker, 2003, 147-158.

[9] G. Di Blasio, K. Kunisch and E. Sinestrari, Stability for abstract linear differential equations, Israel J. Math. 50 (1985), 231-263.

[10] O. Diekmann, S. A. van Gils, S. M. Verduyn Lunel and H.-O. Walther, Delay Equations. Functional, Complex and Nonlinear Analysis, Springer-Verlag, Berlin, 1995.

[11] B. D. Doytchinov, W. J. Hrusa and S. J. Watson, On perturbations of differentiable semigroups, Semigroup Forum 54 (1997), 100-111.

[12] B. Eberhardt, O. El-Mennaoui and K.-J. Engel, On a class of differentiable semigroups, Tübinger Berichte 4 (1994/95), 27-32.

[13] K.-J. Engel and R. Nagel, One-Parameter Semigroups for Linear Evolution Equations, Springer-Verlag, Berlin, 2000.

[14] J. K. Hale and S. M. Verduyn Lunel, Introduction to Functional-Differential Equations, Springer-Verlag, Berlin, 1993.

[15] E. Hille, On the differentiability of semi-group operators, Acta Sci. Math. (Szeged) 12B (1950), 19-24.

[16] L. Maniar and J. Voigt, Linear delay equations in the $L^{p}$ context, in: Evolution Equations, G. R. Goldstein, R. Nagel and S. Romanelli (eds.), Marcel Dekker, 2003, 319-330.

[17] J. Martinez and J. M. Mazon, $C_{0}$-semigroups norm-continuous at infinity, Semigroup Forum 52 (1996), 213-224.

[18] R. Nagel and S. Piazzera, On the regularity properties of perturbed semigroups, Rend. Circ. Mat. Palermo (2) Suppl. 1998, 99-110.

[19] A. Pazy, On the differentiability and compactness of semi-groups of linear operators, J. Math. Mech. 17 (1968), 1131-1141.

[20] A. Pazy, Semigroups of Linear Operators and Applications to Partial Differential Equations, Springer-Verlag, Berlin, 1983.

[21] R. S. Phillips, Perturbation theory for semi-groups of linear operators, Trans. Amer. Math. Soc. 74 (1953), 199-221.

[22] M. Renardy, On the stability of differentiability of semigroups, Semigroup Forum 51 (1995), 343-346.

[23] E. Sinestrari, A noncompact differentiable semigroup arising from an abstract delay equation, C. R. Math. Rep. Acad. Sci. Canada 6 (1984), 43-48.

[24] G. F. Webb, Functional differential equations and nonlinear semigroups in $L^{p}$-spaces, J. Differential Equations 29 (1976), 71-89. 
[25] J. Wu, Theory and Applications of Partial Functional-Differential Equations, SpringerVerlag, Berlin, 1996.

[26] K. Yosida, On the differentiability of semi-groups of linear operators, Proc. Japan Acad. 34 (1958), 337-340.

[27] K. Yosida, Math. Reviews 20 (1959), \#5435. 
